\title{
Noise acceptance of acoustic sequences for indoor soundscape in transport hubs
}

\author{
Bin Wang ${ }^{1}$, Jian Kang ${ }^{1,2}$, Wei Zhao ${ }^{1\left(^{*}\right)}$ \\ 1, School of Architecture, Harbin Institute of Technology; Key Laboratory of Cold Region Urban and Rural Human
}

Settlements Environment Science and Technology, Ministry of Industry and Information Technology, No.66, Xidazhi

Street, Harbin 150001, China

2, Institute for Environmental Design and Engineering, the Bartlett, University College London, London WC1H

ONN, United Kingdom

* Corresponding author.

Abstract: Noise acceptance is an aspect of indoor soundscape research. While staying or walking in a transport hub, sequential sounds form sequence sounds sessions, which is referred to as an acoustic sequence. The basic phenomenon and effects of acoustic sequences on acceptance evaluations have been explored. A total of 209 sections of $30 \mathrm{~s}$ acoustic units were extracted before performing acceptance evaluations. The acoustic units were divided into strong, medium, and weak levels and compiled into 37 pieces of acoustic sequences, which were then subjected to four tests for acceptance evaluation: sound content, acceptance level, and effects of weak and strong acceptance units. One piece of an acoustic sequence consists of 20 acoustic units. The results show that all acoustic sequences exhibit "attenuation effects," as for any acoustic unit that make up the sequence, general acceptance decreases with time. The lower the acceptance, the faster the decay in score. High acceptance units have an "enhancement effect," and the acceptance increases after a high acceptance unit. Low acceptance units have a "boost effect," and the acceptance increases after a low-level acceptance. Both enhancement and boost effects could improve the acceptance evaluation of acoustic sequences and sound experiences in transport hubs.

Keywords: indoor soundscape; acoustic sequence; noise acceptance; transport hubs

Date Received: 10 May 2019

Date Accepted: 17 Dec 2019 
Publish Online: 22 Jan 2020

\section{Introduction}

Environmental noise is unnecessary and harmful, and it typically originates from human activities. It is, therefore, important to effectively evaluate its acceptance or annoyance, which describes the subjective perception of noise ${ }^{1}$, particularly in urban areas where environmental noise levels can be high. Noise acceptance is influenced by a large number of factors, which can be divided into two aspects: 1) acoustic and physical factors and 2) social, psychological, and economic factors. Regarding acoustic and physical factors, Schultz ${ }^{2}$ discussed the relationship between the overall sound pressure level and annoyance, and Hall et al ${ }^{3}$ proposed that noise evaluation depends on the type of noise. Ochiai et $a l^{4}$ indicated that substantial pure tone would increase annoyance based on the spectrum characteristics, and low-frequency components are significant. It was suggested by Fields ${ }^{5}$ that annoyance would not increase after noise events exceeded a certain number. Meanwhile, Griffiths et $a l^{6}$ noted that annoyance in summer is more serious than that in winter, and that at night is more serious than that during the day. In addition, Fields et $a 1^{7}$ proposed that evaluations are also influenced by variations of noise status. As for social, psychological, and economic factors, Nelson ${ }^{8}$ proposed six factors that influence evaluations, whereas Guski ${ }^{9}$ considered that noise annoyance was only related to a third of noise parameters. Asakura et $a l^{10}$ explored the relationship between vision and hearing, noting that vision affects acoustic annoyance evaluations. Noise acceptance and annoyance are both used for noise evaluation. The effects of age ${ }^{11}$, gender ${ }^{12}$, education ${ }^{13}$, behavioral habits ${ }^{14}$, and region ${ }^{15}$ on noise evaluations have also been considered.

Soundscape research began in the 1960s; studies mainly focused on the relationship among human ears, users' characteristics, acoustic environment, and society ${ }^{16}$. For example, several scholars subjectively evaluated sound based on distance, movement, quantity, openness, and duration of sound sources ${ }^{17-18}$. Other studies have revealed the relationship between interviewees' factors and acoustic environment ${ }^{19}$, and the influence of other physical factors on acoustic comfort ${ }^{20}$. A large number of studies have been conducted on indoor soundscape such as underground spaces, hospitals ${ }^{21-22}$, schools ${ }^{23-24}$,restaurants ${ }^{25-26}$ and transport hubs ${ }^{27-28}$. 
Environmental noise is also widespread in transport hubs; that is, urban complex buildings based on transport functions that integrate commerce, accommodation, and recreation, as well as many other functions ${ }^{29}$. The importance of transport hubs has been increasingly recognized ${ }^{30}$. Compared with other building types, they exhibit clear features of spatial combination and human traffic flows ${ }^{31}$, as well as different functional zones with different acoustic environments ${ }^{32}$. Wang and $\mathrm{Kang}^{33}$ proposed the contextualized acoustic environment, termed the "acoustic scene," rather than space, as the basic unit of acoustic perception. The experience of a user who stays within or passes through the environment to form a serialized acoustic scene is termed as "acoustic sequence."

Mathematically, a "sequence" refers to objects, information, or events that are arranged in line; thus, the order of elements is critical. The concept of a space sequence is also significant to architecture and landscape design. Qian ${ }^{34}$ discussed a basic spatial sequence model. Based on the principle of visual perception organization, Sun ${ }^{35}$ discussed the relationship between basic units in the spatial sequence of architecture. From the perspective of visual perception, Liu and Zhang ${ }^{36}$ proposed three elements of landscape spatial sequence: spatial variation, temporal variation, and emotional variation. Furthermore, Jiang ${ }^{37}$ discussed planning and designing strategies from a sequential perspective. Both the spatial sequence and the visual sequence are significant; moreover, noise acceptance research also involves various aspects of auditory sense based on different contexts ${ }^{20}$. Moreover, the concept of acoustic sequence offers an important and novel perspective for soundscape, as the effects of one part of a sound section might differ for different acoustic sequences.

The main aims of this study are therefore to examine the basic phenomena with sound contents and acceptance levels, and study how the effects of acoustic units with different levels of noise acceptance influenced evaluations. Four tests are conducted on acoustic sequences for acceptance evaluation. First, the basic phenomenon of an acoustic sequence is investigated for different sound contents. Second, the basic phenomenon of an acoustic sequence is investigated for different acceptance levels. Third, the effect of acoustic units with high acceptance levels on acoustic sequences is determined. Finally, the effect of acoustic units with low acceptance levels on acoustic sequences is determined. 


\section{Methodology}

The evaluation of an indoor soundscape acoustic sequence is different from that of an acoustic sequence, which is important to focus on changes of evaluation. Therefore, it is necessary to evaluate an acoustic unit independently and put it in the acoustic sequence for comparison. The method has three steps. Firstly, $30 \mathrm{~s}$ acoustic units are collected and extracted from surveys. Secondly, all the acoustic units are evaluated independently to obtain the scores for separate units, when there is no effect on other acoustic unit. Thirdly, pieces of acoustic sequences are formed by acoustic units and noise acceptance is evaluated to obtain the scores for sequences, when there is some influence by adjacent units. The basic phenomenon and effects of acoustic sequences on acceptance are investigated by comparing the scores of separate units and sequences.

\subsection{Collection and extraction of acoustic units}

Six functional types and 30 acoustic scenes have been classified in a transport hub ${ }^{33}$, as detailed in Table I. In this study, original recording data of acoustic scenes in 9 airports, 14 railway stations, 4 bus stations, and 7 subway stations (a total of 34 transport hubs) were collected. Regarding noise measurement, as this study predominantly deals with the temporal domain of sound, the binaural effect and directivity of sound and other issues are not discussed in detail. Therefore, a handheld acoustics and vibration analyzer (BSWA801) connected to a recorder was used to collect the sound data. Continuous recordings were made, with 44100$\mathrm{Hz}$ sampling, 16-Bit quantization, and digital file storage in wav format. Finally, more than $110,000 \mathrm{~s}$ of recording data were obtained.

According to the sound evaluation standard Listening Test on Loudspeakers ${ }^{38}$, the recording duration for subjective assessment is generally 20-40 s. In a transport hub, a complete broadcast is generally 20-30 s, which represents a long-duration acoustic event. Therefore, by setting the time of the acoustic scene unit to $30 \mathrm{~s}$, defined as the "acoustic unit," complete broadcasts can be included, as well as other acoustic events (such as human behavioral sounds and vehicle sounds), which are generally less than $30 \mathrm{~s}$.

To form acoustic sequences, representative acoustic units were extracted from collected recordings. The collected recordings were divided into multiple sections of 30-s-long acoustic units. If the time interval of an acoustic event was too long, the background noise was cut. If some recordings were still too long, the recordings were divided into multiple sections of 30- 
s-long acoustic units. If the content of some samples were similar, one section of the acoustic unit was selected as a representative section. A total of 209 sections of representative acoustic units were extracted, each measuring $30 \mathrm{~s}$.

\subsection{Acoustic units graded}

Both one-dimensional methods (such as ranking) and multiple-dimensional methods (such as pair-wise comparisons and quantized scoring) can be used for noise evaluation ${ }^{1}$. To discuss the principle of the acoustic unit and acoustic sequence, quantized scoring was selected in this study. According to VDI 2563-Scale ${ }^{39}$, a quantized score ranging from 1 to 10 indicated the influence of noise from "strong" to "weak." In ISO/TS 15666: 2003(E) ${ }^{40}$, a 0-to-10 opinion scale indicated "not at all annoyed" to "extremely annoyed." Based on these specifications, a quantized score of 1 to 10 was used, which indicated "not accepted at all" to "extremely accepted."

TABLE I Different function types and acoustic scenes used in the experiments

\begin{tabular}{|c|c|c|c|}
\hline $\begin{array}{l}\text { Function } \\
\text { type }\end{array}$ & Acoustic scene & $\begin{array}{l}\text { Function } \\
\text { type }\end{array}$ & Acoustic scene \\
\hline \multirow{2}{*}{$\begin{array}{l}\text { 1. Transport } \\
\text { transfer }\end{array}$} & \multirow{2}{*}{$\begin{array}{l}\text { 1. City street } \\
\text { 2. Passage outside station } \\
\text { 3. Station concourse } \\
\text { 4. Bus station } \\
\text { 5. Taxi station } \\
\text { 6. Platform of rail transit } \\
\text { 7. Other parks }\end{array}$} & $\begin{array}{l}\text { 3. In-station } \\
\text { traffic }\end{array}$ & $\begin{array}{l}\text { 15. In-station passage } \\
\text { 16. In-station hall } \\
\text { 17. Platform passage } \\
\text { 18. Platform }\end{array}$ \\
\hline & & \multirow[b]{2}{*}{$\begin{array}{l}\text { 4. Transport } \\
\text { auxiliary }\end{array}$} & $\begin{array}{l}\text { 19. Ticket area } \\
\text { 20. Check-in area } \\
\text { 21. Baggage area }\end{array}$ \\
\hline \multirow{3}{*}{ 2. Vehicles } & \multirow{2}{*}{$\begin{array}{l}\text { 8. Train carriage } \\
\text { 9. Cabin } \\
\text { 10. Coach carriage } \\
\text { 11. Rail carriage } \\
\text { 12. Bus carriage }\end{array}$} & & $\begin{array}{l}\text { 22. Inspection area } \\
\text { 23. Ticket checking area } \\
\text { 24. Area of inquiry and } \\
\text { exhibition }\end{array}$ \\
\hline & & $\begin{array}{l}\text { 5. Station } \\
\text { waiting area }\end{array}$ & $\begin{array}{l}\text { 25. Large waiting area } \\
\text { 26. Medium and small } \\
\text { waiting area }\end{array}$ \\
\hline & $\begin{array}{l}\text { 13. Taxi carriage } \\
\text { 14. Other vehicles }\end{array}$ & $\begin{array}{l}\text { 6. Living } \\
\text { auxiliary }\end{array}$ & $\begin{array}{l}\text { 27. Toilet } \\
\text { 28. Dining area } \\
\text { 29. Commercial area } \\
\text { 30. Other auxiliary area }\end{array}$ \\
\hline
\end{tabular}

The subjective evaluation of sound is influenced by different ages, and young people 
have highly sensitive hearing ${ }^{11}$. According to previous studies, around 20 subjects are relatively common for simple listening evaluations. Seven subjects with normal hearing were selected to complete a soundscape test in Liu and Kang's research ${ }^{41}$. Furthermore, Hemple and Chouard pointed out that the test results of 20 subjects could meet statistical requirements for an ordinary listening evaluation test ${ }^{42}$. In this study, only one indicator of acceptance was evaluated, and the listening tests were relatively simple. Additionally, only the average score of acceptance was employed, and no demographic or social factors were analyzed. Thirty participants were randomly selected from the list of 1,100 students at School of Architecture in Harbin Institute of Technology. The selected participants were asked whether or not they would like to take part in the tests, and they all accepted. The 30 subjects were between 20 and 28 years old, with 16 males and 14 females. They were trained and pilot tests were conducted to ensure the stability for evaluations. Other groups of ages were not included in this study. The evaluation was conducted at an audiometric laboratory of the Architectural Science and Engineering Research Center of Cold Area.

Before formal evaluation, pilot training was conducted to make the subjects understand the listening process and evaluation method. Because 209 acoustic units were independent of each other, according to the feedback of the subjects in the pilot training, it was determined that continuous experiments should be followed by rest for $15 \mathrm{~min}$ after every $15 \mathrm{~min}$, with the training being conducted for no more than $3 \mathrm{~h}$ per day. In the formal evaluation, all 209 acoustic units were listened to one by one. When one acoustic unit with $30 \mathrm{~s}$ was heard, a pause of $15 \mathrm{~s}$ was used to evaluate noise acceptance. The purpose of the pause was to reduce the influence of adjacent acoustic units.

All 30 participants evaluated 209 acoustic units. The inter-rater reliability, $0.813-0.911$ (Cronbach's $\alpha$ ), was acceptable. The split-half method was used to further verify the score of each group, and the Spearman-Brown coefficient was between 0.801 and 0.942 . Therefore, the average score of all 30 participants for each acoustic unit was employed as the initial score. The score of each acoustic unit represents the average experience to the individual section of sound.

To detect the basic phenomenon of acoustic sequences with acceptance changes, the 209 acoustic units were divided into three levels according to the initial scores. Based on VDI 2563-Scale and ISO/TS 15666: 2003(E), an acoustic unit with a score of 7 or more is Level I, 
indicating a high acceptance unit. A score from 4 to 7 corresponds to Level II, indicating a medium acceptance unit, and a score of less than 4 corresponds to Level III, indicating a low acceptance unit.

\subsection{Evaluation of acoustic sequences}

Pilot tests were performed for evaluating acoustic sequences, including the number of acoustic units that composed the sequences, evaluation method, types and adjustments of sequences.

The first pilot test determined the number of acoustic units that composed the sequences. A total of 20,40, and 60 sections of acoustic units have been tried respectively to compose a piece of sequence. The results were basically consistent. Additionally, continuous listening should not exceed 30 min based on the feedback of the subjects. Consequently, 20 acoustic units, which consisted of one piece of a sequence, are sufficient and appropriate. All acoustic sequences are composed of 20 sections of 30-s-long acoustic units in a certain order.

The second pilot test was the evaluation method. In order to explore the evaluation changes of acoustic units in sequences and the influence of each other unit, 20 acoustic units in one acoustic sequence would be evaluated 20 times in turn, rather than evaluating them only once at the end of the sequence. Similarly, a VDI scale was used to evaluate the noise acceptance of acoustic sequences. Moreover, it is found that there was a $3 \mathrm{~s}$ interval between the sound units, which was helpful for evaluating the acceptance at the end of the sound units and record the score. As a result, each acoustic sequence needs approximately $11 \mathrm{~min}$ and must be evaluated continuously. Two acoustic sequences can be evaluated within $30 \mathrm{~min}$, followed by rest for $15 \mathrm{~min}$, with the tests being conducted for no more than $3 \mathrm{~h}$ per day.

The third pilot test was performed to determine the type of acoustic sequence and adjustment. The evaluation of noise acceptance of acoustic sequences was categorized into Level I, Level II, and Level III, similar to that of VDI. In actual acoustic environments, the acoustic characteristics are not always continuous. Therefore, characteristics of continuity, cyclicity and abrupt changes have been considered for the tests. The acoustic characteristics for Test 1 are continuous and cyclical. The acoustic characteristics of contents for Test 2 are varied (including abrupt change and discontinuity), with 20 different acoustic units. Based on Test 2, Test 3 and Test 4 were carried out to examine the effect of different levels on annoyance in a wider range. 
As mentioned above, four formal tests for evaluating noise acceptance on acoustic sequences were conducted, as detailed in Fig. 1. One piece of an acoustic sequence was composed of 20 acoustic units, while each unit was set to $30 \mathrm{~s}$, and a short pause for $3 \mathrm{~s}$ was set between adjacent units. Participants could listen to the acoustic sequence and evaluate the acceptance during the pause. It meant that one piece of acoustic sequence needed nearly 11 minutes. All acoustic sequences are composed in a certain order.

\subsubsection{Test 1 - Evaluation on acoustic sequences of content}

Test 1 was conducted to indicate the relationship between acoustic content and sequence, which is the basic phenomenon of acoustic sequence. Acoustic units with Levels I, II, and III were selected respectively from six functional types in transport hubs, which are mentioned in Table I. As a result, a total of 18 acoustic units were selected in Test 1. One acoustic unit was $30 \mathrm{~s}$ long and was played 20 times repeatedly, with a $3 \mathrm{~s}$ pause between each acoustic unit, so as to form one piece of an acoustic sequence. Thus, there were 18 pieces of acoustic sequences in Test 1.

Participants could listen to the acoustic sequence and evaluate the acceptance during the pause. As a result, the curve of 20 scores for one sequence was obtained, which are the "sequence scoring curves" shown in Fig. 2. A total of 18 "sequence scoring curves" were obtained in Test 1. This could help in studying the relationship between acoustic content and sequence.

\subsubsection{Test 2 - Evaluation on acoustic sequences with acceptance levels}

Test 2 was conducted to indicate the effect of acceptance levels on acoustic sequence. Twenty Level I acoustic units were selected, as an example. The acoustic units were arranged with scores from low to high, to form an acoustic sequence in this test, and a curve of "unit original scores" was obtained. A unit with the highest score of 8.13 was selected at the end of the sequence, to detect whether the highest acceptance unit would improve sequence evaluations at the end in the sequence.

Participants listened to the acoustic sequence and evaluated acceptance, as mentioned above. After evaluation, the average score of each acoustic unit in sequence was calculated and a curve of "sequence scores" was obtained. The differences between those two curves represent changes that indicate the effect of acceptance level on acoustic sequences. Level II and Level III sequences were composed and evaluated in the same way as Level I units in this 
test.

\subsubsection{Test 3 - Effects of substituted high acceptance units}

Test 3 was conducted to determine the effects of high acceptance units on acoustic sequences, which exhibiting medium and low acceptance. Based on Test 2, an acoustic unit with a score of 7.73, which was a Level I unit, had high acceptance, and ranked 95\% from low to high (the highest score was 8.13), was selected to replace another unit in acoustic sequences, which were Level II and Level III sequences in Test 2, respectively, by comparing the effects in different ways.

In the 20 Level II acoustic sequence, as an example, the start sound section (120-150 s), the middle section (270-300 s), and the end section (420-450 s) were replaced respectively and simultaneously with a Level I acoustic unit having a score of 7.8. Four substituted modes were used to compose the four acoustic sequences of Level II for evaluation. After evaluation, the curves of scores for replaced sequences were obtained. Comparisons are conducted among these four substituted curves, a curve of "unit original scores" and a curve of "sequence scores" for Level II sequence in Test 2, which indicate the effect of high acceptance unit on acoustic sequences. Level III sequences were composed and evaluated in the same way as Level II sequence in this test.

\subsubsection{Test 4 - Effects of substituted low acceptance units}

Test 4 was conducted to determine the effects of low acceptance units on acoustic sequences, which exhibited high and medium acceptance. Based on Test 2, an acoustic unit with a score of 2.17, which was a Level III acoustic unit, had low acceptance, and ranked 5\% from low to high (the lowest score was 1.77), was selected to replace another unit in acoustic sequences, which were Level I and Level II sequences in Test 2, respectively, by comparing the effects in different ways. Four substituted modes, for Level I and Level II sequences, respectively, were composed and evaluated in the same way as that in Test 3.

Generally speaking, a piece of an acoustic sequence was composed of 20 acoustic units, while each unit was set to $30 \mathrm{~s}$, and a short pause of $3 \mathrm{~s}$ was set between adjacent units. It meant that one piece of acoustic sequence needed nearly 11 minutes. There are a total of 37 pieces of acoustic sequences for all four tests: 18 pieces in Test 1, 3 pieces in Test 2, 8 pieces in Test 3, and 8 pieces in Test 4 . Thirty subjects, as mentioned above, were employed to evaluate the noise acceptance for acoustic sequences. Each participant needed to take a rest 
after evaluating one piece of sequence. Up to nine pieces of sequences were evaluated per day. Therefore, Test 1 took two days and Tests 2 to 4 took one day, respectively, for each participant.

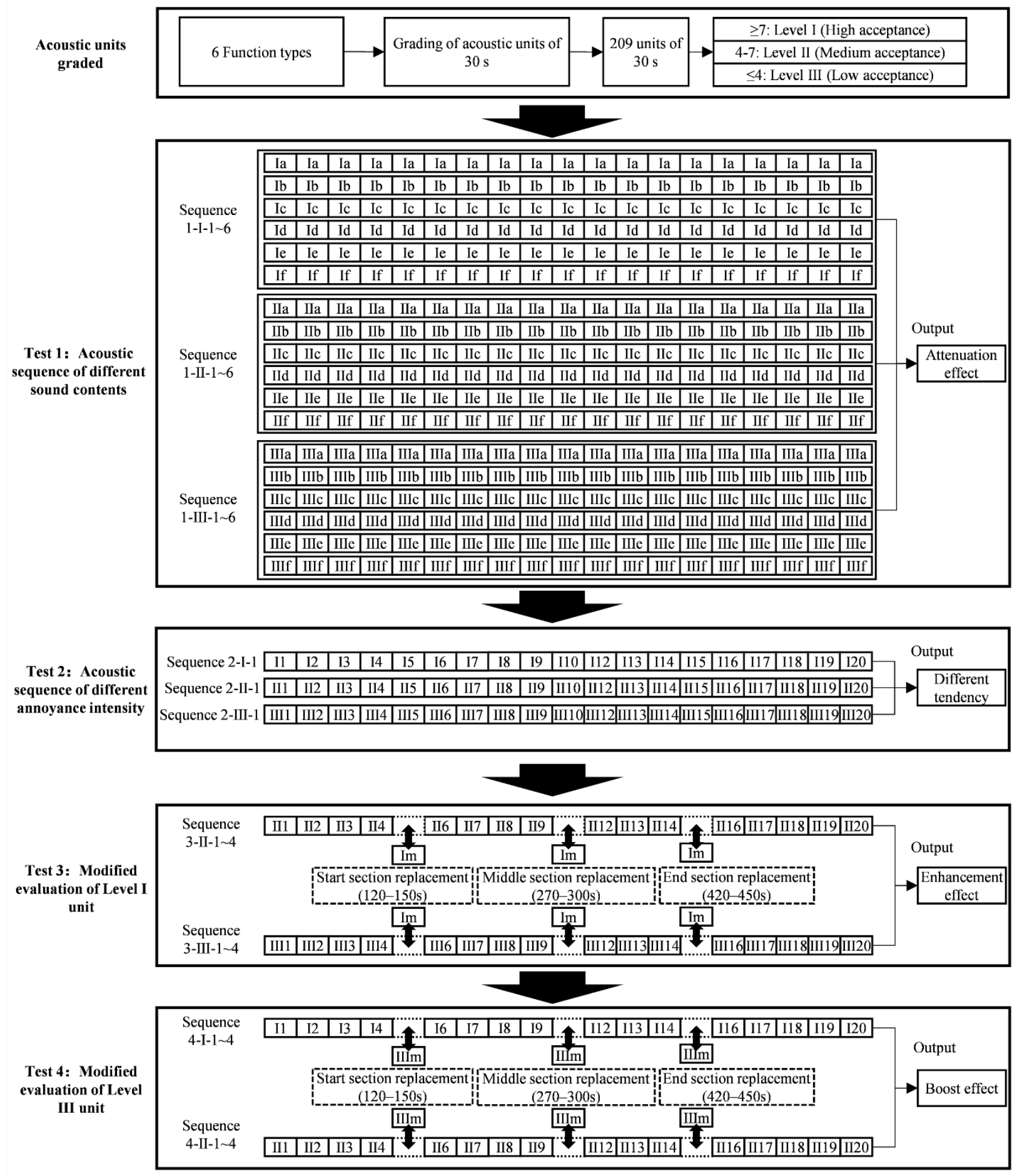

Fig. 1 Framework of the test process 


\section{RESULTS}

\subsection{Acoustic sequence changes for different sound contents}

In Test 1, the evaluations of the acoustic sequences representing six types of sound content were compared for the three acceptance levels, as shown in Fig. 2. Despite some fluctuations, the overall trend is a decrease of acceptance with time, which is known as the "attenuation effect". The variation of the end points of the total 18 acoustic sequences for Test 1 are described in Table II. For Level I units, as shown in Fig. 2a and Table II, the attenuation trend of sequences is approximately linear, and the difference in scores between the start points and end points of sequence varies from 0.67 to 1.93 , decreasing from $8.3 \%$ to $24.7 \%$. For Level II units, as shown in Fig. 2b and Table II, the score differences vary from 2 to 2.77, decreasing from $39 \%$ to $58.9 \%$. The decreasing score of acceptance given to the acoustic sequence is particularly clear for the station waiting unit, especially with increased time. For Level III units, as shown in Fig. 2c, the acceptance scores decrease to zero (minimum acceptance) at 330-540 s. The attenuation is most rapid for the station waiting unit. However, the attenuation of the vehicle acoustic sequence is the slowest. These results also indicate that the subjects could clearly identify different acoustic sequences and make distinct evaluations, which are similarly to the previous research that people were able to recognize the type of space in train stations just by listening to its soundscape ${ }^{27}$. The attenuation effect appears to be a basic phenomenon for acoustic sequences, which is independent of sound contents. That is, for the same sound, the longer it lasts, the lower the acceptance.

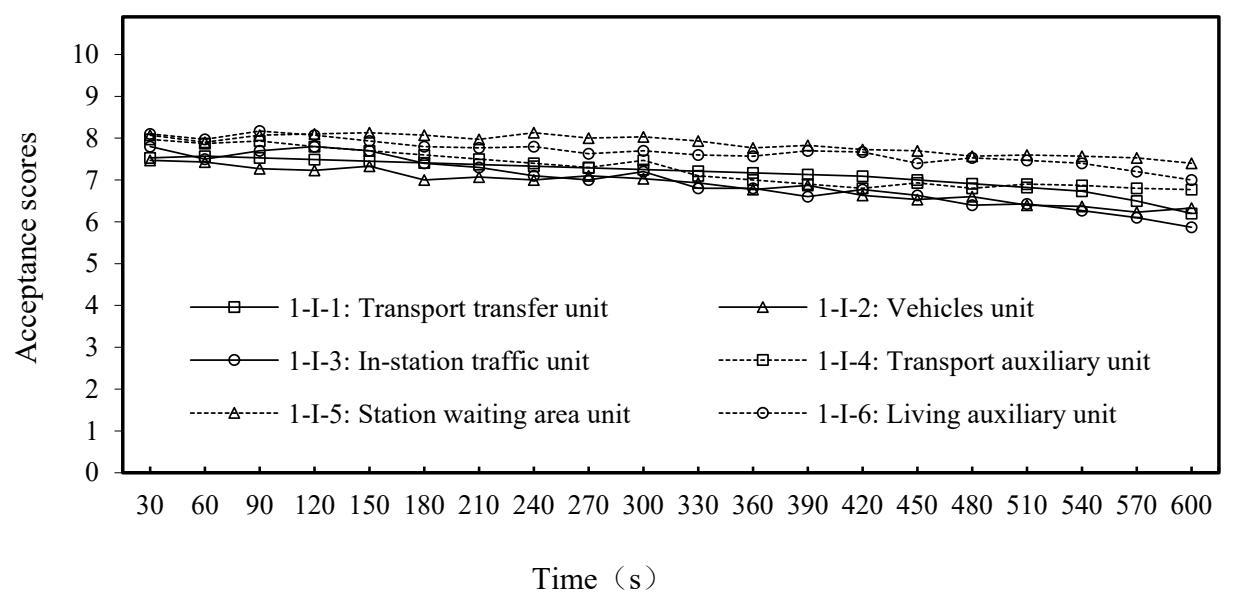


(a) Level I

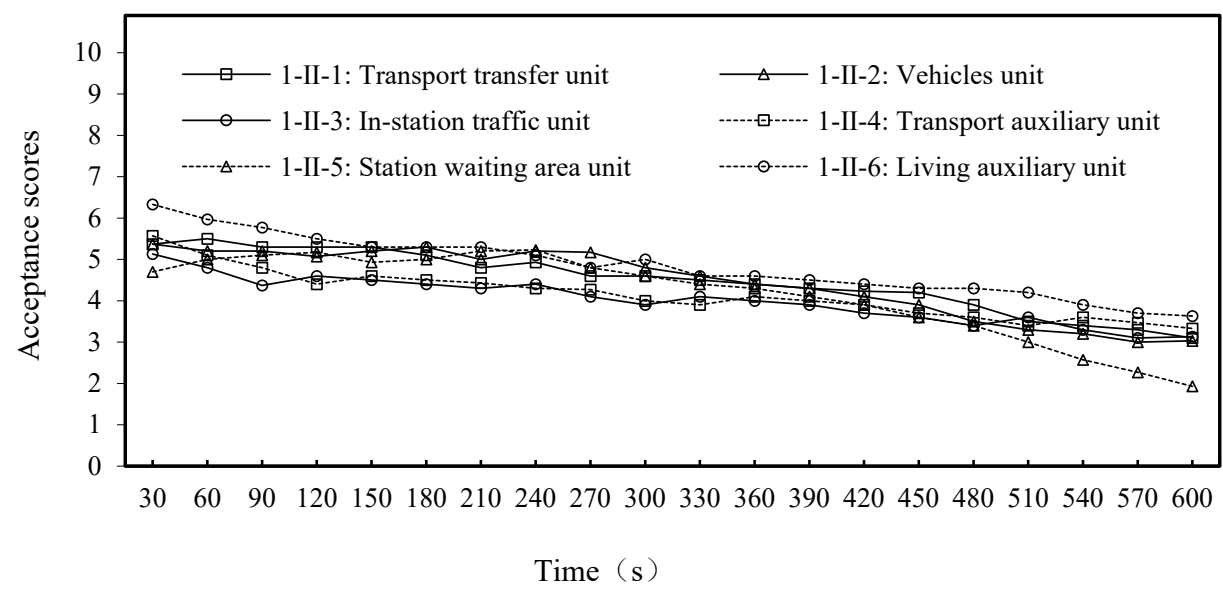

(b) Level II

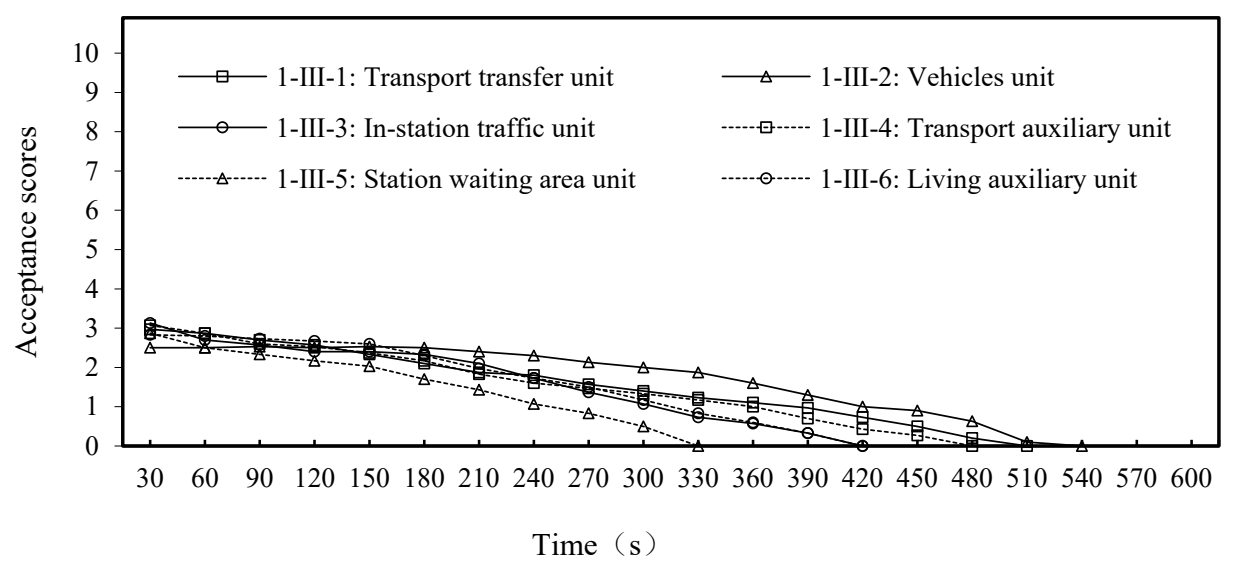

(c) Level III

Fig. 2 Cyclic evaluation of the acoustic units

\subsection{Evaluation of acoustic sequences with different acceptance levels}

The evaluations of acoustic sequences with different acceptance levels are compared in Fig. 3. The score at the starting point of the acoustic sequence only differs slightly from the initial score of the start unit; therefore, the score at the end point of the sequence are compared with the initial score of the end unit, as listed in Table II. For Level I units, as shown in Fig. $3 \mathrm{a}$, the acoustic unit with the initial highest score of 8.13 was arranged at the end of the Level I acoustic sequence. However, after evaluating the sequence, the score of the end unit in the acoustic sequence is 6.53 . The distance between the two curves increases, indicating that the score gradually decreases. The score is approximately linear but slowly attenuates by $19.7 \%$ at the end of the sequence. For Level II units, as shown in Fig. 3b, the initial score for the acoustic unit is 6.93 , but it was only 2.47 for the acoustic sequence. The overall score shows 
a steady decline, with substantial fluctuations. Due to a large decrease with time, the attenuation is $64.4 \%$ at the end of the sequence. For Level III, as shown in Fig. 3c, Level III acoustic units with an end score of 3.97 were selected; yet, the end scores of the acoustic sequences are 0 . The test was terminated at $510 \mathrm{~s}$, where the recorded attenuation is $100 \%$. These results illustrate that the attenuation speed differs considerably with the acceptance intensity of the acoustic sequence. The lower the acceptance of the acoustic sequence, the faster the attenuation of the acceptance evaluation scores.

TABLE II Comparisons of scores at the ends of all sequences

\begin{tabular}{|c|c|c|c|c|c|c|c|}
\hline $\begin{array}{l}\text { Acoustic } \\
\text { sequences }\end{array}$ & $\begin{array}{l}\text { Scores at } \\
\text { the start of } \\
\text { acoustic } \\
\text { sequences }\end{array}$ & $\begin{array}{l}\text { Scores at } \\
\text { the end of } \\
\text { acoustic } \\
\text { sequences }\end{array}$ & Changes & $\begin{array}{c}\text { Acoustic } \\
\text { sequences }\end{array}$ & $\begin{array}{l}\text { Scores at the } \\
\text { end for no } \\
\text { adjustment }\end{array}$ & $\begin{array}{l}\text { Scores at the } \\
\text { end for } \\
\text { adjustments }\end{array}$ & Changes \\
\hline Test 1 & & & & Test 3 & & & \\
\hline $1-\mathrm{I}-1$ & 7.53 & 6.2 & $-17.7 \%$ & 3 -II-1 & 2.47 & 3.67 & $48.6 \%$ \\
\hline $1-\mathrm{I}-2$ & 7.47 & 6.33 & $-15.3 \%$ & $3-\mathrm{II}-2$ & 2.47 & 4.77 & $93.1 \%$ \\
\hline $1-I-3$ & 7.8 & 5.87 & $-24.7 \%$ & $3-\mathrm{II}-3$ & 2.47 & 4.3 & $74.1 \%$ \\
\hline $1-\mathrm{I}-4$ & 7.97 & 6.77 & $-15.1 \%$ & $3-\mathrm{II}-4$ & 2.47 & 5.5 & $122.7 \%$ \\
\hline $1-\mathrm{I}-5$ & 8.07 & 7.4 & $-8.3 \%$ & 3-III-1 & 0 & 0 & - \\
\hline $1-\mathrm{I}-6$ & 8.1 & 7 & $-13.6 \%$ & 3-III-2 & 0 & 1.4 & - \\
\hline $1-\mathrm{II}-1$ & 5.37 & 3.1 & $-42.3 \%$ & 3-III-3 & 0 & 0.63 & - \\
\hline 1-II-2 & 5.37 & 3.03 & $-43.6 \%$ & 3-III-4 & 0 & 2.53 & - \\
\hline $1-\mathrm{II}-3$ & 5.13 & 3.13 & $-39.0 \%$ & Test 4 & & & \\
\hline $1-\mathrm{II}-4$ & 5.57 & 3.33 & $-40.2 \%$ & $4-I-1$ & 6.53 & 5.83 & $-10.7 \%$ \\
\hline $1-\mathrm{II}-5$ & 4.7 & 1.93 & $-58.9 \%$ & $4-\mathrm{I}-2$ & 6.53 & 6.77 & $3.7 \%$ \\
\hline 1-II-6 & 6.33 & 3.63 & $-42.7 \%$ & $4-\mathrm{I}-3$ & 6.53 & 7.33 & $12.3 \%$ \\
\hline 1-III-1 & 2.97 & 0 & $-100 \%$ & $4-\mathrm{I}-4$ & 6.53 & 6.47 & $-0.9 \%$ \\
\hline 1-III-2 & 2.5 & 0 & $-100 \%$ & 4 -II-1 & 2.47 & 2.03 & $-17.8 \%$ \\
\hline 1-III-3 & 3.13 & 0 & $-100 \%$ & 4-III-2 & 2.47 & 5.43 & $119.8 \%$ \\
\hline 1-III-4 & 3.07 & 0 & $-100 \%$ & $4-\mathrm{II}-3$ & 2.47 & 4.63 & $87.4 \%$ \\
\hline $1-\mathrm{III}-5$ & 2.87 & 0 & $-100 \%$ & $4-\mathrm{II}-4$ & 2.47 & 3.77 & $52.6 \%$ \\
\hline 1-III-6 & 2.83 & 0 & $-100 \%$ & & & & \\
\hline $\begin{array}{c}\text { Acoustic } \\
\text { sequences }\end{array}$ & $\begin{array}{c}\text { Scores at } \\
\text { the end of } \\
\text { acoustic } \\
\text { units }\end{array}$ & $\begin{array}{l}\text { Scores at } \\
\text { the end of } \\
\text { acoustic } \\
\text { sequences }\end{array}$ & Changes & & & & \\
\hline \multicolumn{8}{|l|}{ Test 2} \\
\hline $2-I$ & 8.13 & 6.53 & $-19.7 \%$ & & & & \\
\hline $2-\mathrm{II}$ & 6.93 & 2.47 & $-64.4 \%$ & & & & \\
\hline 2-III & 3.97 & 0.00 & $-100 \%$ & & & & \\
\hline
\end{tabular}




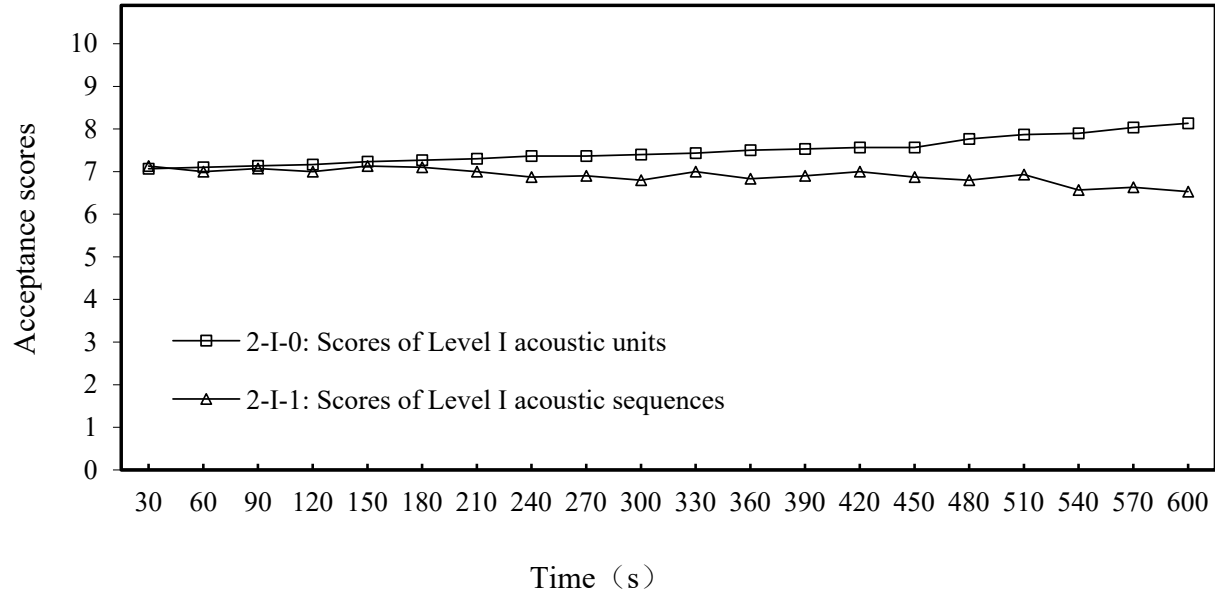

(a) Level I

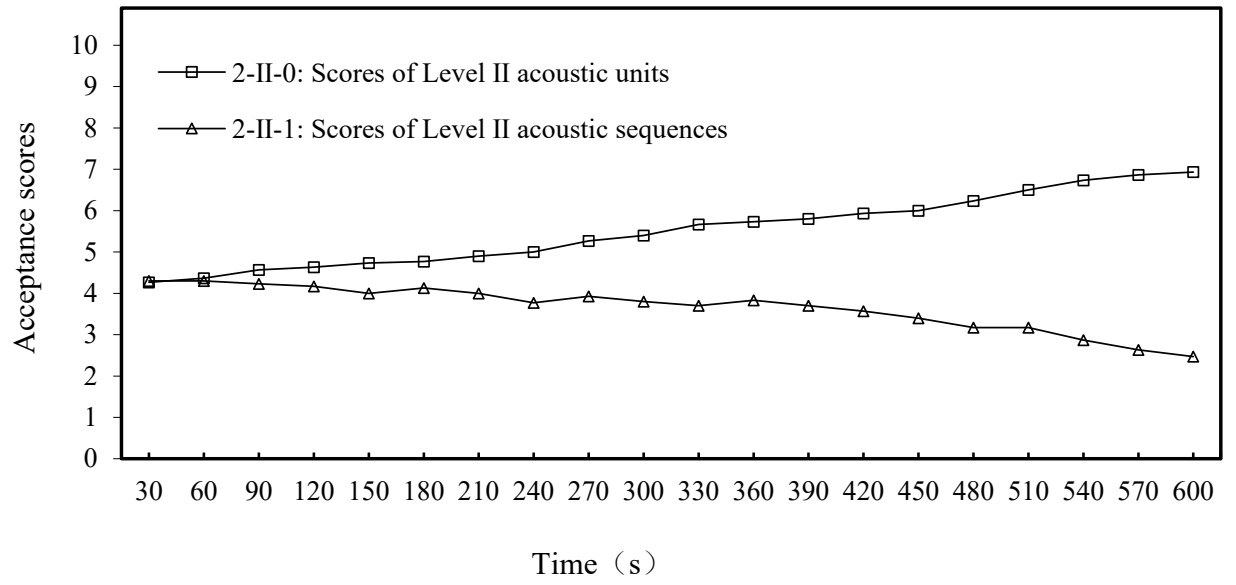

(b) Level II

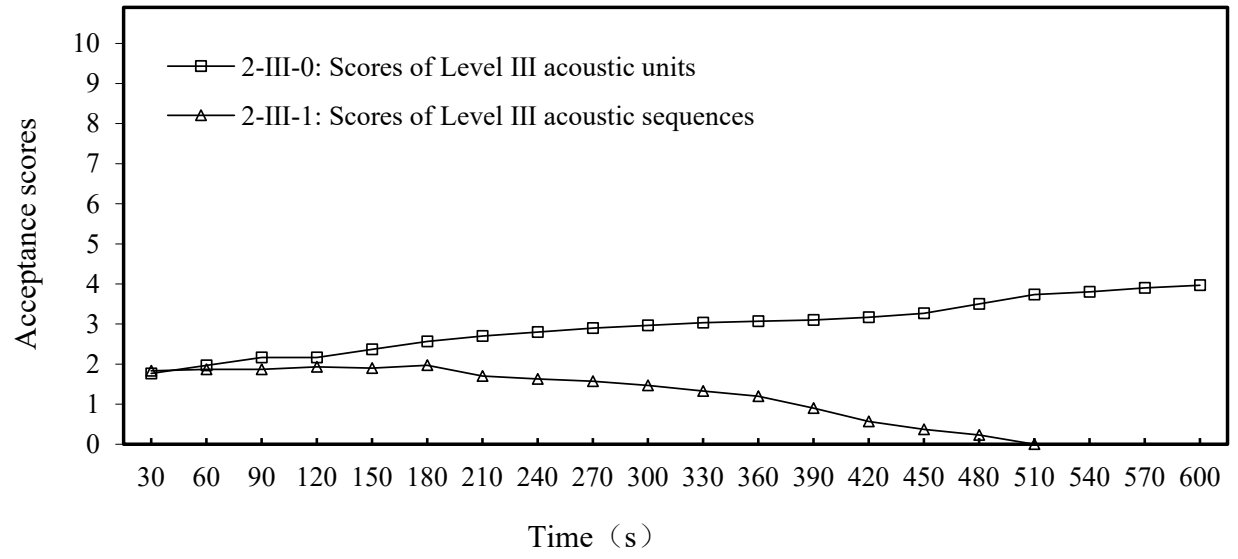

(c) Level III

Fig. 3 Accumulative evaluation of the acoustic sequences 


\subsection{Modified effect of acoustic units with high acceptance on acoustic sequences}

The modified effects of acoustic units with high acceptance on other acoustic sequences are illustrated in Fig. 4. Comparisons of the effects with adjustments are listed in Table II. The acoustic unit with initial scores of 6.93 and acoustic sequences with evaluated scores of 2.47 (as mentioned in Test 2 from Level II) are compared with modified acoustic sequences in Fig. 4a. The scores at the end of the acoustic sequence are 5.5 for modification involving all three sections (three-section modification), an increase of $122.7 \% ; 4.77$ for middle-section modification, an increase of $93.1 \%$; 4.3 for end-section modification, an increase of $74.1 \%$; and 3.67 for start-section modification, an increase of $48.6 \%$.

Furthermore, the score for the middle-section modification increases sharply to a peak of 8.27, then decreases to 5.07 for the acoustic unit with an initial score of 5.67 at $330 \mathrm{~s}$, which indicates that there is a psychological gap between high score units (high acceptance) and low score units (low acceptance). The score for end-section replacement is 0.47 lower than that for middle-section replacement, which is mainly due to the "attenuation effect," as illustrated by Fig. 2. After another 7 mins of attenuation then replacement, it does not achieve a good result. Replacement of the start section also improves the overall score by 1.2 ; however, after the early start-section replacement, the modification effect is reduced, which is also primarily due to long-time attenuation.

Acoustic units with initial scores of 3.97 and acoustic sequences from Level III with evaluation scores of 0 are compared with modified sequences in Fig. $4 \mathrm{~b}$. The end scores of the acoustic sequence are 2.53 for three-section modification, 1.4 for middle modification, 0.63 for end modification, and 0 for start modification; thus, three-section modification has the best effect of reducing scores.

In terms of middle-section modification, the score increases by 1.4 because the Level III acoustic sequence is cut into two sections of approximately $5 \mathrm{~min}$, each of which still reaches the accelerated attenuation area. In terms of end-section modification, the average score of acoustic units is 7.13 , which is less than the 7.73 score for the initial acoustic unit. Furthermore, the attenuation is accelerated after it is modified with the end section, exhibiting the fastest attenuation of evaluation scores. This also indicates that, if an acoustic sequence with low acceptance is modified too late, the effect will be reduced. In terms of start-section modification, the average score of acoustic units is 8.47 ; therefore, the scores of the latter 
section are also increased, but the attenuation continues and finally becomes 0 at $540 \mathrm{~s}$, which indicates that an acoustic sequence of Level III with low acceptance should not be overly long.

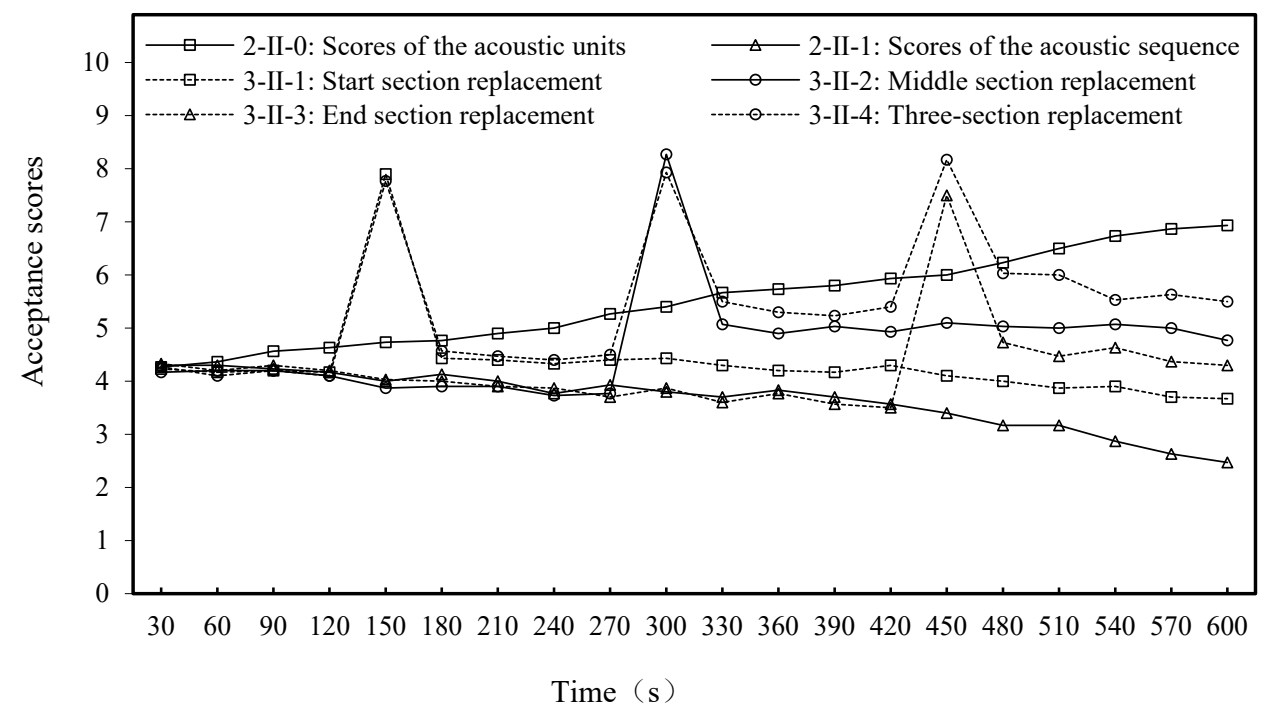

(a) Level II

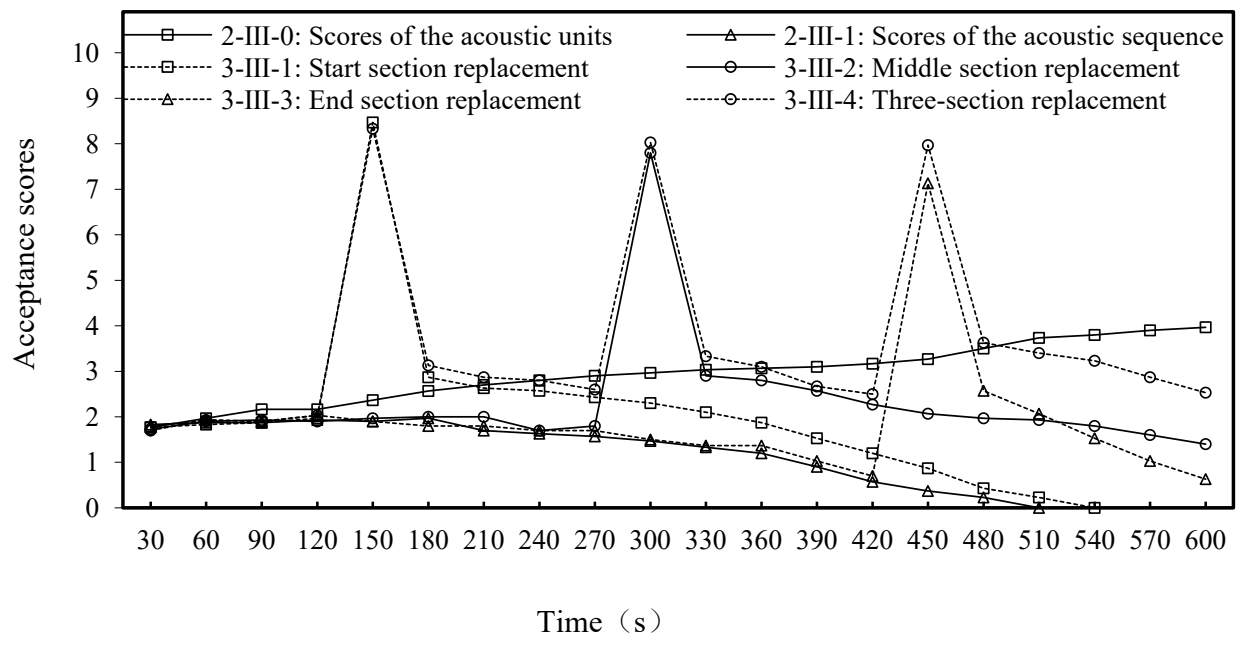

(b) Level III

Fig. 4 Modification of Level I acoustic units to the acoustic sequences

These results illustrate that Level I acoustic units with high acceptance have a positive influence on acceptance scores, which is defined as the "enhancement effect" for acoustic sequences. All alternatives improve the scores of the acoustic sequences, which significantly affect noise acceptance evaluations. Moreover, a similar study on high acceptance of acoustics on evaluation was conducted in a previous research, in which the pleasantness of traffic noise 
environments increases significantly, when the occurrence of birdsong over a period of $30 \mathrm{~s}$ is increased $^{43}$, and water sound resulted in an improvement of environmental noise, where the perception of annoyance reduced and that of pleasantness increased ${ }^{44}$.

\subsection{Modified effect of acoustic units with low acceptance on acoustic sequences}

The modified effects of acoustic units with low acceptance on other acoustic sequences are illustrated in Fig. 5. Comparisons of the effects with adjustments are listed in Table II. Acoustic units with initial scores of 8.13 and acoustic sequences with evaluated scores of 6.53 (as mentioned in Test 2 from Level I) were compared with modified acoustic sequences. The end score of the acoustic sequence was 7.33 for end-section modification, an increase of $12.3 \%$; 6.77 for middle-section modification, an increase of $3.7 \% ; 6.47$ for three-section modification, a decrease of $0.9 \%$; and 5.83 for start-section modification; a decrease of $10.7 \%$. Compared to other types of modification, end-section modification shows the best effect on acceptance scores, with slow attenuation from a high starting point of 7.87 at $480 \mathrm{~s}$ after 7 min of attenuation.

Furthermore, with middle-section modification, the score increases back to 7.73 at $330 \mathrm{~s}$; however, the acoustic unit of Level III will also have a negative influence, leading to subsequently rapid attenuation. The effect of three-section modification is not the worst, although acoustic units of Level III were added into the acoustic sequence of Level I. This is mainly because acoustic units are replaced many times in the acoustic sequence, leading to rich sound experience for the subjects. Thus, start-section modification exhibits the worst effect. Long-term attenuation results in the lowest score at the maximum duration, although this score increases after an early modification.

Fig. 5b compares acoustic units with initial scores of 6.93 and acoustic sequences from Level II with evaluation scores of 2.47 with modified sequences. Table II summarizes the comparisons of the effects with adjustments. The end scores for the acoustic sequence are 5.43 for middle-section modification, an increase of $119.8 \%$; 4.63 for end-section modification, an increase of $87.4 \% ; 3.77$ for three-section modification, an increase of $52.6 \%$; and 2.03 for start-section modification, a decrease of $17.8 \%$. Among all types of modification, middlesection modification shows the best effect, with an improvement of $119.8 \%$; the boost effect greatly improves the score at the starting point to even higher than that of the initial acoustic unit. The attenuation during the first $5 \mathrm{~min}$ is approximately the same as that of cumulative 


\section{evaluation.}

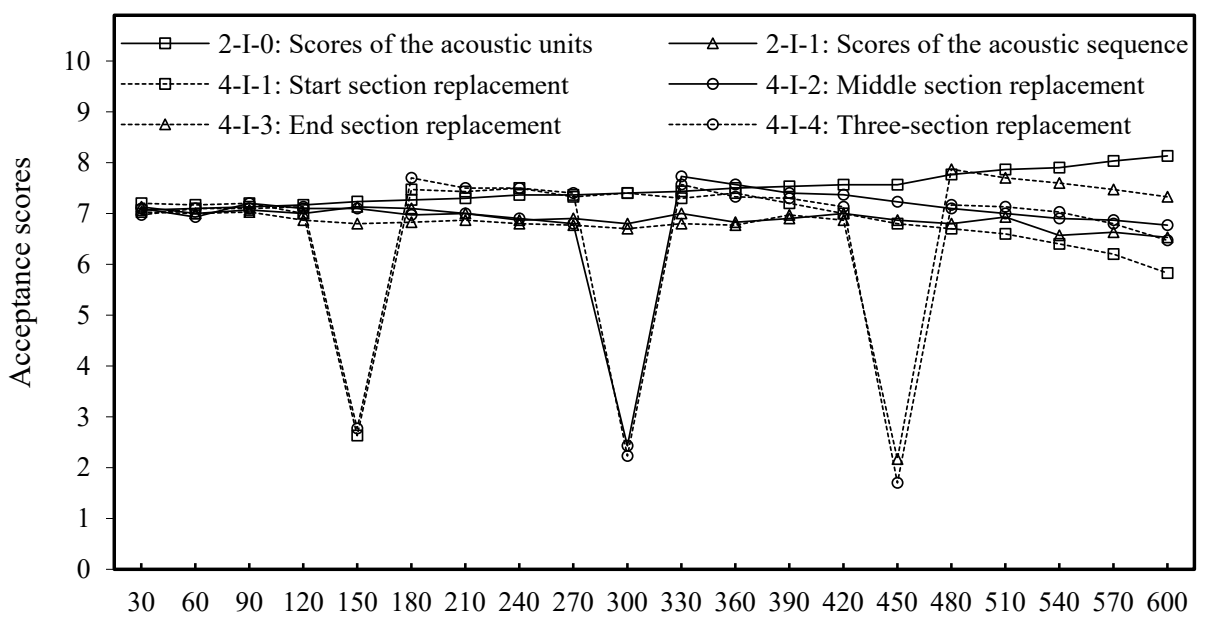

Time $(s)$

(a) Level II

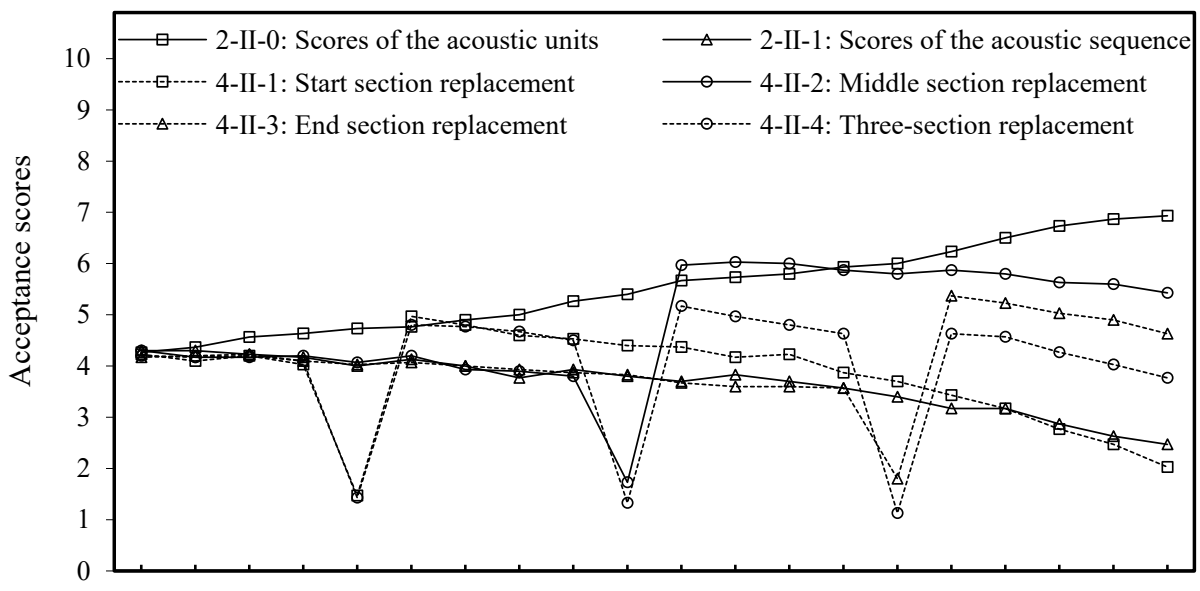

$30 \quad 60 \quad 90120150180210240270300330360390420450480510540570600$

Time（s）

(b) Level III

Fig. 5 Modification of Level III acoustic units to the acoustic sequences

Furthermore, with end-section modification, the score is attenuated to a low value after $7 \mathrm{~min}$ of decay. The boost effect is not as obvious as that for middle-section modification. With three-section modification, only the score of the first acoustic unit replaced is higher than that of initial unit, and then the boost effect is weak. With start-section modification, after a small increase in the score, it gradually decays to 0.44 less than the cumulative scores. 
Those results illustrate that the acoustic unit of Level III units with low acceptance have a negative influence on acceptance evaluation scores. However, the score boosts after a Level III unit, which is termed the "boost effect" of the acoustic sequence. It indicates that the appropriate use of Level III units can improve the overall score of the acoustic sequence, and then soundscape, although it was pointed out that noise in stations would reduce the acoustic comfort $^{28}$ in a previous study.

\section{CONCLUSIONS}

Various acoustic scenes in a transport hub were investigated, and representative acoustic units were selected. Acceptance evaluations of the acoustic units were conducted and divided into three levels: high, medium and low acceptance. Based on their different functions, four groups of tests with acoustic sequences were evaluated. The evaluations involved different sound contents, different acceptance intensities, and the modified effects of high and low acceptance units. This study found that, for young people aged from 20 to 28 , the acceptance evaluation decays with time, regardless of the type of acoustic sequence. The duration of the acoustic sequence varies with different acceptance levels. Furthermore, enhancement effect and boost effect can be used to improve the acceptance evaluation of acoustic sequences and sound experiences in a transport hub. The following conclusions were made:

1. All acoustic sequences exhibit an attenuation effect. This study indicates that the annoyance accumulates over time, shown by the attenuation trend, regardless of the acoustic sequences with sound content, and even displays some fluctuations.

2. The lower the acoustic acceptance, the faster the attenuation of the acceptance evaluation. The longer the duration of the low acceptance sequence, the faster the attenuation. Quick attenuation begins at 2-3 min. The decay of the high acceptance sequence is the slowest, but the duration cannot be too long.

3. An acoustic unit with high acceptance has an enhancement effect, which could improve the acoustic sequence, and the effects differ greatly. In terms of medium acceptance sequences, the effect of three-section modification is optimal. An acoustic unit with high acceptance could be used to replace the acoustic unit every $3 \mathrm{~min}$. The effect of middle-section modification is better, corresponding to a high acceptance unit replaced at $5 \mathrm{~min}$. In terms of low acceptance sequences, the effect of three-section modification 
is the best, corresponding to a 3-min replacement with a weak acoustic unit.

4. An acoustic unit with low acceptance exhibits a boost effect, which could be properly utilized to improve the acoustic sequence. Modification with the low acceptance unit leads to a boost of the scores after a substantial decrease. For acoustic sequences with a high acceptance, the effect of end-section replacement is the best, corresponding to a low acceptance unit at a 7-8 min replacement position. For acoustic sequences with medium acceptance, the effect of middle-section modification is significantly better, corresponding to replacement at $5 \mathrm{~min}$.

Based on these conclusions, some examples of acoustic design strategies in transport hubs can be proposed, with initial evidence based on the age group of 20 to 28 . Waiting areas in transport hubs could be divided into rest areas and transit areas. Rest areas could be differentiated using the time spent there, and the acoustic environment should be actively modified at specific time intervals. In a large waiting hall, when there are few trains, it will be quiet for a long time, representing a high acceptance of acoustic sequence, but eventless and boring over time. Based on findings in Test 4, an acoustic unit of medium or low acceptance, such as an advertisement, could be displayed every 7-8 min to adjust the acoustic sequence to increase the level of acceptance. In a long and noisy corridor, based on findings in Test 3 , the acoustic environment could be improved by arranging sound absorbers including plants at specific places ${ }^{45-47}$, as this would lead to short acoustic sequences that should improve acceptance. For the same long corridor, if there are few people, it would be quiet all the time, and acoustic units with low acceptance such as advertisements could be played halfway along the corridor to enrich the sequence and improve the acceptance. In general, the longer the acoustic sequence, the greater the annoyance. Therefore, recommendations include the wide use of medium acceptance acoustic sequences, limiting the duration of low acceptance sequences, and enriching strong acceptance sequences to improve the acoustic environment. Furthermore, the evaluations on acoustic sequences for the different characteristics of people such as ages, gender, and cultural background are discussed for future works.

\section{Acknowledgements}

This work is supported by the National Key R\&D Program of China (Grant 
No.2016YFC0700700), the China Postdoctoral Science Foundation (2017M621278) and the European Research Council Advanced Grant (No. 740696).

\section{References}

1 C. Marquisfavre, E. Premat, and D. Aubrée, "Noise and its effects - a review on qualitative aspects of sound. Part II: Noise and annoyance," Acta Acust. Acust. 91(4), 613$625(2005)$.

2 T. J. Schultz, "Synthesis of social surveys on noise annoyance," J. Acoust. Soc. Am. 65(3), 377-405 (1978).

3 F. L. Hall, S. E. Birnie, S. M. Taylor, and J. E. Palmer, "Direct comparison of community response to road traffic noise and to aircraft noise," J. Acoust. Soc. Am. 70(6), 1690-1698 (1981).

${ }^{4}$ H. Ochiai, Y. Tokita, and S. Yamada, "Study on evaluation method of infra and low frequency noise," Fort Lauderdale, December 06-08, International Congress and Exhibition on Noise Control Engineering, USA (1999).

5 J. M. Fields, "The effect of numbers of noise events on people's reactions to noise: an analysis of existing survey data," J. Acoust. Soc. Am. 75(2), 447-467 (1984).

6 I. D. Griffiths, F. J. Langdon, and M. A. Swan, "Subjective effects of traffic noise exposure: reliability and seasonal effects," J. Sound Vib. 71(2), 227-240 (1980).

7 J. M. Fields, R. G. de Jong, A. L. Brown, I. H. Flindell, T. Gjestland, R. F. S. Job, S. Kurra, P. Lercher, A. Schuemer-Kohrs, M. Vallet, and T. Yano, "Guidelines for reporting core information from community noise reaction surveys," J. Sound Vib. 206(5), 685-695 (1997).

8 J. P. Nelson, “Airports and property values: a survey of recent evidence," J. Transp. Econ. Policy. 14(1), 37-52 (1980).

9 R. Guski, "Personal and social variables as co-determinants of noise annoyance," Noise Health. 1(3), 45-56 (1999).

10 T. Asakura, S. Tsujimura, M. Yonemura, L. Hyojin, and S. Sakamoto, "Effect of immersive visual stimuli on the subjective evaluation of the loudness and annoyance of 
sound environments in urban cities," Appli. Acoust. 143, 141-150 (2019).

11 R. Rylander, S. Sörensen, and A. Kajland, “Annoyance reactions from aircraft noise exposure," J. Sound Vib. 24(4), 419-444 (1972).

12 A. M. Verzini, C. A. Frassoni, and A. H. Ortiz, "A field study about the effects of low frequency noise on man," J. Acoust. Soc. Am. 105(2), 942-942 (1999).

13 J. M. Fields, "Effect of personal and situational variables on noise annoyance in residential areas," J. Acoust. Soc. Am. 93(5), 2753-2763 (1993).

14 P. Lercher, "Deviant dose-response curves for traffic noise in 'sensitive areas,," Christchurch, November 16-18, International Congress and Exhibition on Noise Control Engineering, USA (1998).

15 T. Gjestland, "Regional differences in noise annoyance assessments," Christchurch, November 16-18, International Congress and Exhibition on Noise Control Engineering, USA (1998).

16 R. M. Schafer, "Our sonic environment and the soundscape: The tuning of the world," Rochester - Destiny Books, (1994).

17 B. Berglund, Ö. Axelsson, and M. E. Nilsson, "The soundscape explicated," Arch. Acoust. 30, Supplement, 127-130 (2005).

18 J. Kang and M. Zhang, "Semantic differential analysis of the soundscape in urban open public spaces," Build. Enviro. 45, 150-157 (2010).

19 J. Hatfield, I. V. Kamp, and R. F. S. Job, “Clarifying 'soundscape': Effects of question format on reaction to noise from combined sources," Acta Acust. United Ac. 92, 922-928 (2006).

20 J. Kang, "Urban sound environment," London - Taylor \& Francis incorporating Spon, (2006).

21 X. Qin, J. Kang, and H. Jin, "Sound environment of waiting areas in large general hospitals in China," Acta Acust. United Ac. 98, 760-767 (2012).

22 G. Watts, A. Khan, and R. Pheasant, "Influence of soundscape and interior design on anxiety and perceived tranquility of patients in a healthcare setting," Appli. Acoust. 104, 135-141 (2016).

${ }^{23}$ H. Xie, J. Kang, and R. Tompsett, "The impacts of environmental noise on the academic 
achievements of secondary school students in Greater London,” Appli. Acoust. 72, 551-555 (2011).

24 D. M. Connolly, J. E. Dockrell, B.M. Shield, R. Conetta, and T. J. Cox, "Students' perceptions of school acoustics and the impact of noise on teaching and learning in secondary schools: findings of a questionnaire survey,” Energ. Procedia 78, 3114-3119 (2015).

25 X. Chen and J. Kang, “Acoustic comfort in large dining spaces,” Appli. Acoust. 115, 166$172(2016)$.

26 Q. Meng, S. Zhang, and J. Kang, "Effects of typical dining styles on conversation behaviours and acoustic perception in restaurants in China," Build. Environ. 121, 148-157 (2017).

27 J. Tardieu, P. Susini, F. Poisson, P. Lasareff, and S. McAdams, "Perceptual study of soundscapes in train stations," Appli. Acoust. 69, 1224-1239 (2008).

28 P. Wang, Y. Wang, C. Zou, and J. Guo, “A preliminary investigation of noise impact within metro stations in the urban city of Guangzhou, China,” Environ. Sci. Pollut. Res. 24, 11371-11382 (2017).

29 S. Li and Q. Zhang, "High-speed rail transportation hub development mode: from transport complex to urban complex," Plann. 07, 26-30 (2011) (in Chinese).

30 Y. Cheng, "The urban design integration to transportation hub renovation oriented - the comprehensive transportation hub of Tianjin station area as example," Master dissertation, Tianjin Univ., Tianjin, 2007 (in Chinese).

31 D. Chen, "Mega rail transit complex in Shanghai Hongqiao transport hub," Time Archit. 05, 38-43 (2009) (in Chinese).

32 H. Pu, X. Qiu, and J. Wang, “Acoustic field boundaries of coupled volumes with different energy decay styles," Acta Acustica (Chinese edition). 34, 533-538 (2009) (in Chinese).

33 B. Wang and J. Kang, "The construction of the sound environment perception theory of traffic complex based on the grounded theory," Urban. Archit. 225(10), 118-120 (2016) (in Chinese).

34 C. Qian, "Study on space sequence antetype," Master dissertation, Zhejiang Univ., Hangzhou, 2003 (in Chinese). 
35 Y. Sun, "A research on the relationships of spatial sequence based on visual perception theory," Master dissertation, Zhejiang Univ., Hangzhou, 2011 (in Chinese).

36 B. Liu and T. Zhang, "Landscape space sequence organization based on visual sense," Chinese Lands. Archit. 26(11), 31-35 (2010) (in Chinese).

37 H. Jiang, "Study on spatial sequence of principal axis landscape of university campus," Master dissertation, Southeast Univ., Nanjing, 2017 (in Chinese).

38 GB/T12060.13-2011. Sound system equipment - part 13: listening test on loudspeakers (Chinese National Standard, Beijing, 2011) (in Chinese).

39 Bestimmen der geraeuschanteile von strassenfahrzeugen mit verbrennungskraftmaschine. Vdi-richtlinie. (Determination of noise components of road vehicles with combustion engines.) VDI guideline. 2563 (1970).

40 Acoustics - Assessment of noise annoyance by means of social and socio-acoustic surveys. ISO/TS 15666:2003(E). (2003).

41 J. Liu, J. Kang, H. Behm, and T. Luo, "Effects of landscape on soundscape perception: soundwalks in city parks," Landsc. Urban Plan. 123, 30-40 (2014).

42 T. Hempel and N. Chouard, "Evaluation of interior car sound with a new specific semantic differential design,” J. Acoust. Soc. Am. 105(2), 1280-1285 (1999).

43 Y. Hao, J. Kang, and H. Wörtche, "Assessment of the masking effects of birdsong on the road traffic noise environment," J. Acoust. Soc. Am. 140, 978-987 (2016).

44 W. Yang, H. J. Moon, and M. J. Kim, "Perceptual assessment of indoor water sounds over environmental noise through windows," Appli. Acoust. 135, 60-69 (2018).

45 J. Kang, "A method for predicting acoustic indices in long enclosures," Appli. Acoust. 51(2), 169-180 (1997).

46 H. Yang, J. Kang, and C. Cheal, "Random-incidence absorption and scattering coefficients of vegetation," Acta Acust. United Ac. 99, 379-388 (2013).

47 J. Kang, "The unsuitability of the classic room acoustical theory in long enclosures," Archit. Sci. Rev. 39, 89-94 (1996). 